\title{
Rapid Clearance of a Structural Isomer of Bilirubin during Phototherapy
}

John F. Ennever, Andrew T. Costarino, Richard A. Polin, and William T. Speck

Department of Pediatrics, Case Western Reserve University School of Medicine, Rainbow Babies and Children's Hospital, Cleveland, Ohio 44106; and Department of Pediatrics, University of Pennsylvania School of Medicine, Division of Neonatology,

Children's Hospital of Philadelphia, Philadelphia, Pennsylvania 19104

\begin{abstract}
During phototherapy for neonatal jaundice, bilirubin is converted into a variety of photoproducts. Determination of the relative importance of these photoproducts to the elimination of bilirubin requires knowledge of their rates of excretion. We have measured the rate at which the structural isomer of bilirubin, lumirubin, disappeared from the serum of nine jaundiced premature infants after the cessation of phototherapy. In all patients studied, the decline in serum lumirubin could be approximated by a firstorder rate equation with a half-life of 80 to $158 \mathrm{~min}$. This rate of disappearance is much faster than that previously determined for the other major bilirubin photoproducts. In samples of bile aspirated from the duodenum of infants undergoing phototherapy, lumirubin was the principal bilirubin photoproduct found. These results indicate that formation and excretion of lumirubin is an important route for bilirubin elimination during phototherapy.
\end{abstract}

\section{Introduction}

Hyperbilirubinemia is one of the most prevalent medical disorders of newborn infants, and affects $25-50 \%$ of all neonates and an even higher percentage of prematurely born infants (1). Since the association between high plasma levels of bilirubin and brain damage is well documented, the management of hyperbilirubinemia is one of the more common problems in neonatal medicine. Visible light phototherapy is an effective (2) and frequently used (3) treatment for neonatal hyperbilirubinemia. Exposure to visible light causes the transformation of unconjugated (indirect-reacting) bilirubin from a lipid-soluble, unexcretable form to more polar isomers and fragments that are eliminated by the liver and kidney. Three types of photochemical reactions of bilirubin occur during phototherapy: configurational isomerization, structural isomerization, and photooxidation (4, 5). It has been assumed that the fastest reaction, configurational isomerization, is quantitatively the most important pathway for the elimination of bilirubin during phototherapy (6-8). This has been shown for pigment elimination in the phototherapy-treated adult Gunn rat, an animal with life-long unconjugated hyperbilirubinemia (9). However, we have recently demonstrated that in human infants the excretion of the configurational isomers

This paper was presented in part at a meeting of the Society for Pediatric Research, May 1985.

Address reprint requests to Dr. Ennever, Dept. of Pediatrics, Rainbow Babies and Children's Hospital, 2101 Adelbert Rd., Cleveland, OH 44106.

Received for publication 5 February 1986 and in revised form 2 December 1986

J. Clin. Invest.

(C) The American Society for Clinical Investigation, Inc.

$0021-9738 / 87 / 06 / 1674 / 05 \$ 1.00$

Volume 79, June 1987, 1674-1678 is too slow for this pathway to be the major route of bilirubin elimination (10). In this study we present evidence that excretion of the structural isomer of bilirubin, lumirubin, is an important, and perhaps primary, pathway by which pigment is eliminated during phototherapy.

\section{Methods}

Patients. The subjects of this study were nine premature infants with physiological hyperbilirubinemia who were admitted to the Intensive Care Unit of The Children's Hospital of Philadelphia. The characteristics of the study population are shown in Table I. All infants were nursed naked and supine on radiant warmer beds and received mechanical ventilation for respiratory distress syndrome. Informed consent was obtained from each patient's parent in accordance with the guidelines established by the hospital's Committee for the Protection of Human Subjects. Decisions on the initiation and termination of phototherapy were based on standard clinical criteria. Phototherapy was administered continuously with broad-spectrum (white) light from a 250-W tungsten-halogen lamp (type ENH; General Electric Co., Cleveland, $\mathrm{OH}$ ) in a Cavitron model PT 1400 phototherapy unit which contains a dichroic filter to remove infrared irradiation (an emission spectrum of the lamp is given in Fig. 1. of Ennever et al. [11]). Irradiation at the umbilicus was measured with an Air-Shields PR-III radiometer (400-500-nm bandwidth, peak response $450 \mathrm{~nm}$ ), and was maintained between 10 and $14 \mu \mathrm{W} / \mathrm{cm}^{2}$ per $\mathrm{nm}$. After at least $24 \mathrm{~h}$ of continuous phototherapy, a serum sample was obtained and phototherapy was stopped. Five additional serum samples were obtained at 5, 15, 30, 60, and $90 \mathrm{~min}$ after stopping therapy. After the last serum sample was obtained phototherapy was restarted.

Serum bilirubin analysis. Blood samples were obtained through indwelling arterial catheters and were shielded from light during collection. A portion of the initial blood sample was used for determination of total serum bilirubin using an American Optical bilirubinometer. The serum was separated by centrifugation and transferred to foil-wrapped tubes for storage at $-20^{\circ} \mathrm{C}$ before chromatographic analysis. The serum samples were analyzed (without further preparation) by reversed-phase high pressure liquid chromatography (HPLC) on Dupont ODS (C-18) columns equipped with a precolumn, using $0.1 \mathrm{M}$ di- $n$-octylamine acetate in methanol as the mobile phase (5). 10- $\mu$ l samples were injected using the full-injection technique for quantitative reproducibility (12). The effluent from the column was monitored with a Hewlett-Packard model 1040A high-speed scanning detector and the absorption peaks at $450 \mathrm{~nm}$ were integrated with a Hewlett-Packard model 3390A integrator. Control experiments with solutions of bilirubin irradiated in vitro demonstrated that integrated peak areas for lumirubin, $4 Z, 15 E$-bilirubin and native bilirubin were proportional to concentration between 0.04 and $20 \mathrm{mg} /$ $\mathrm{dl}$; all serum samples had bilirubin and bilirubin isomer concentrations that fell within this range. The chromatographic peak assignments were based upon retention times and absorption spectra that were compared with synthetic standards. Because retention times can vary with age of the column, synthetic standards were analyzed daily. The apparent halflife of the structural isomer in the serum was estimated for each patient from the linear least-squares best fit of the log of serum lumirubin concentration vs. time after stopping phototherapy.

Bile analysis. Six of the nine patients had nasojejunal tubes in place for enteral feeding while enrolled in the study. Samples of duodenal bile were obtained by aspiration while these patients were undergoing pho- 


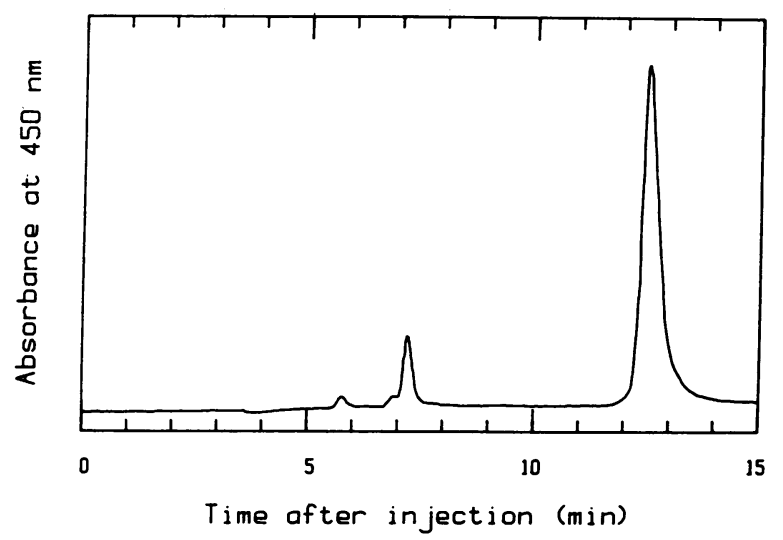

Figure 1. Bilirubin isomers in the serum of a jaundiced infant receiving phototherapy. HPLC shows absorbance at $450 \mathrm{~nm}$ plotted against time after sample injection. The principal peaks are: native $(4 Z, 15 Z)$ bilirubin eluting at $12.7 \mathrm{~min}, 4 Z, 15 E$-bilirubin at $7.2 \mathrm{~min}$ (the $4 E, 15 Z$-isomer is the unresolved component just before the $4 Z, 15 E$ peak), and the structural isomer, lumirubin, at $5.8 \mathrm{~min}$.

totherapy. In addition, bile samples were also obtained before or after phototherapy from three of these patients. These samples were shielded from light during collection and were stored frozen at $-20^{\circ} \mathrm{C}$. Before analysis, bile samples were thawed, briefly centrifuged to pellet any suspended solids, and the undiluted supernatant injected into the HPLC. The HPLC retention times and absorption spectra of mono- and diglucuronide conjugates of bilirubin were determined using fresh bile obtained by bile duct cannulation of an adult Dark Agouti rat (13).

\section{Results}

It has been shown previously that the two major classes of bilirubin photoproducts, configurational and structural isomers, reach steady state levels in the serum within 3 to $4 \mathrm{~h}$ of beginning phototherapy $(10,14)$. A chromatograph of serum obtained just before stopping phototherapy is shown in Fig. 1. The most abundant bilirubin photoproducts are the configurational isomers, $4 Z, 15 E$ - and $4 E, 15 Z$-bilirubin. In human infants, the $4 Z, 15 E$-isomer is the principal configurational isomer found in the serum, presumably as the result of the regioselectivity of the configurational isomerization reaction of bilirubin bound to human albumin (5). The structural isomer, lumirubin, which is formed more slowly than the configurational isomers, is found in far lower concentrations in the serum than the configurational isomers. ${ }^{1}$ In our study population of nine infants, at the time phototherapy was stopped for the disappearance study, the structural isomer lumirubin was $3.2 \pm 1.4 \%( \pm \mathrm{SD})$ of the total pigment in the serum, and the configurational isomers (principally the $4 Z, 15 E$-isomer) were $17.0 \pm 2.1 \%$ of the total.

After the interruption of phototherapy, the concentration of lumirubin in the serum decreases rapidly. Fig. 2 is a plot of the concentration of lumirubin in the serum of one of the patients

1. The relative peak areas from the chromatogram do not accurately reflect the relative molar amounts of each bilirubin isomer present in the sample, because the detector response for the various isomers differs. The relative response ratios at $450 \mathrm{~nm}$ are 1.0:0.70:0.43 for $4 Z, 15 Z$ bilirubin/4Z,15E-bilirubin/lumirubin (15). These corrections (with 0.70 used for the $4 E, 15 Z$-isomer) were used in calculations of the percent composition listed in Table I and the text.

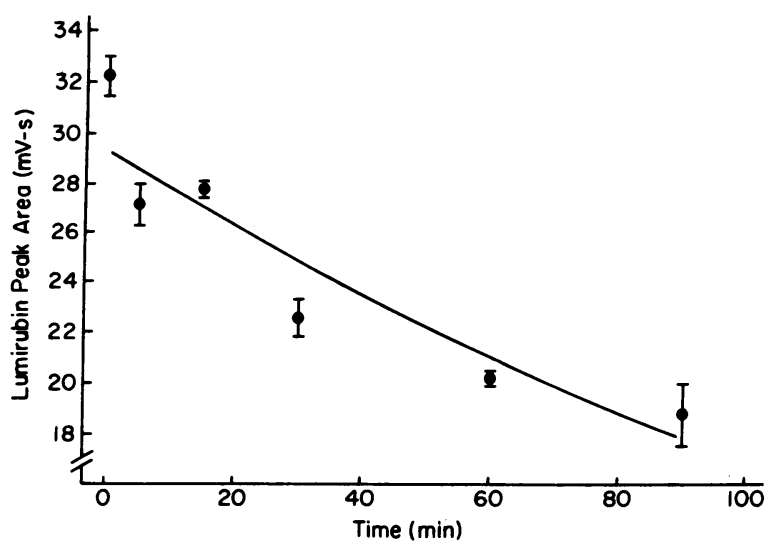

Figure 2. Disappearance of lumirubin from serum of patient three after cessation of phototherapy. Lumirubin peak area from HPLC analysis of serum is plotted as a function of time after stopping phototherapy. The data points are the mean and error bars indicate one standard deviation of two or three chromatographic analyses. The lumirubin peak area is plotted on a linear scale (in units of $\mathrm{mV} \cdot \mathrm{s}$ from the HPLC integrator). The curve is the least-squares best fit of the data to a first-order rate equation.

as a function of time after stopping phototherapy. In all the patients studied, the data on lumirubin concentration as a function of time approximated that expected for first-order kinetics with correlation coefficients ranging from 0.77 to 0.94 (Table I). Using this approximation, the apparent half-life of lumirubin in the serum ranged from 80 to $158 \mathrm{~min}$ with a mean of $112 \mathrm{~min}$.

Lumirubin was the principal isomer of bilirubin found in samples of duodenal bile from infants under phototherapy (Figs. 3 and 4). No lumirubin was found in bile samples obtained before the start of phototherapy (Fig. 3) or $24 \mathrm{~h}$ after ending phototherapy (Fig. 4). Identification of the material eluting at $6.2 \mathrm{~min}$ as lumirubin was based upon chromatographic retention time and absorption spectrum of the material (Fig. 5), both of which were identical to that of lumirubin prepared in vitro as described by McDonagh and co-workers (5). All samples of bile contained $450 \mathrm{~nm}$ absorbing material with chromatographic retention times similar to that of native $(4 Z, 15 Z)$ bilirubin $(\sim 13$ min) and mono- and di-glucuronide conjugates of bilirubin ( $~ 6.9$ and $5.3 \mathrm{~min}$, respectively). ${ }^{2}$ The major yellow pigment found in all samples of bile obtained from infants undergoing phototherapy was lumirubin.

\section{Discussion}

When bilirubin is exposed to light, whether in the test tube, in the Gunn rat, or in the human infant, it is rapidly converted from a nonpolar internally hydrogen bonded structure to a variety of more polar isomeric forms. During phototherapy, this conversion is only the first step in bilirubin elimination; once formed, these polar isomers must be excreted. In the adult Gunn

2. The broad asymmetric chromatographic peaks for the mono- and diglucuronide conjugates of bilirubin are presumably the result of the intramolecular migration of bilirubin from the C-1 to the other three hydroxyl groups of the glucuronic acid (13). 


\begin{tabular}{|c|c|c|c|c|c|c|c|c|c|}
\hline \multirow[b]{2}{*}{ Patient } & \multirow[b]{2}{*}{$\begin{array}{l}\text { Gestational } \\
\text { age }\end{array}$} & \multirow[b]{2}{*}{$\begin{array}{l}\text { Birth } \\
\text { weight }\end{array}$} & \multirow[b]{2}{*}{$\begin{array}{l}\text { Age at start of } \\
\text { phototherapy }\end{array}$} & \multicolumn{4}{|c|}{ At start of disappearance study* } & \multirow{2}{*}{$\begin{array}{l}\text { Apparent } \\
\text { lumirubin } \\
\text { half-life' }\end{array}$} & \multirow[b]{2}{*}{$\begin{array}{l}\text { Correlation } \\
\text { coefficient }\end{array}$} \\
\hline & & & & Age & $\begin{array}{l}\text { Total serum } \\
\text { bilirubin }\end{array}$ & Lumirubin & $\mathbf{Z E}+\mathrm{EZ}^{*}$ & & \\
\hline & $w k$ & $k g$ & $d$ & $d$ & $m g / d l$ & $\%$ & $\%$ & $\min$ & \\
\hline 1 & 27 & 0.91 & 2 & 4 & 4.3 & 2.1 & 18.2 & 104 & 0.80 \\
\hline 2 & 30 & 1.16 & 2 & 3 & 6.0 & 5.4 & 21.2 & 131 & 0.94 \\
\hline 3 & 30 & 1.20 & 1 & 2 & 8.9 & 4.3 & 17.8 & 124 & 0.92 \\
\hline 4 & 31 & 1.42 & 2 & 3 & 11.6 & 2.2 & 13.3 & 103 & 0.91 \\
\hline 5 & 32 & 1.35 & 4 & 5 & 7.0 & 3.4 & 17.2 & 85 & 0.86 \\
\hline 6 & 32 & 1.60 & 4 & 5 & 6.9 & 2.1 & 16.9 & 125 & 0.77 \\
\hline 7 & 32 & 1.70 & 2 & 4 & 8.6 & 4.7 & 17.3 & 158 & 0.87 \\
\hline 8 & 32 & 1.84 & 3 & 4 & 12.0 & 3.7 & 16.2 & 80 & 0.91 \\
\hline 9 & 36 & 2.27 & 4 & 5 & 14.2 & 0.9 & 14.5 & 96 & $0.8 \dot{4}$ \\
\hline
\end{tabular}

* 90-min study period during which phototherapy was stopped began after at least $24 \mathrm{~h}$ of continuous phototherapy. Values listed for total bilirubin (determined spectrophotometrically) and percentage composition (determined by HPLC) are for initial sample obtained immediately after stopping phototherapy. ${ }^{\ddagger}$ Configurational isomers: $4 Z, 15 E$ - and $4 E, 15 Z$-bilirubin, ${ }^{\S}$ Apparent half-life calculated from linear least-squares best fit of experimental data to a first-order rate equation. "Correlation coefficient for log of serum lumirubin concentration vs. time after stopping phototherapy.

rat biliary excretion of both configurational and structural isomers is rapid (9), and since configurational isomerization is by far the faster of the two isomerization reactions, it contributes most to the efficacy of phototherapy in the rat. It has been assumed, until recently, that the faster configurational isomerization reaction was the more important pathway for bilirubin elimination in human infants as well.

Several observations suggested that the excretion of the rapidly formed configurational isomers is extremely slow in human infants. Soon after techniques for measurement of the bilirubin isomers were developed, it was reported that the configurational isomers achieved high steady state levels $(15-20 \%$ of the total bilirubin) in the serum of infants treated with phototherapy $(7$, 8). In premature infants treated with white light phototherapy, the concentration of configurational isomers in the serum is nearly equal to the photoequilibrium level achieved for bilirubin-

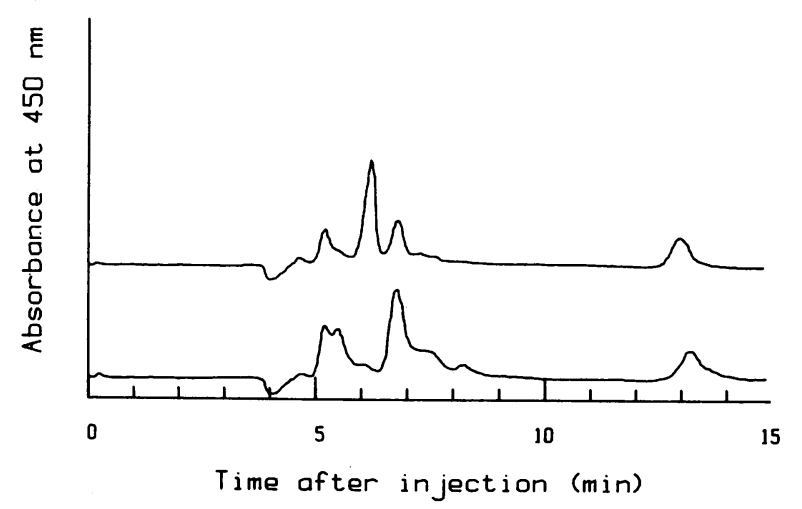

Figure 3. Bilirubin composition in bile of patient number nine. Chromatographic analysis was performed on bile obtained before phototherapy (bottom tracing) and during phototherapy (top tracing). Both chromatograms are at same detector attenuation. The principal peaks are lumirubin $(\sim 6.2 \mathrm{~min})$, native bilirubin $(\sim 13 \mathrm{~min})$, bilirubin diglucuronide ( $\sim 5 \mathrm{~min})$, and bilirubin mono-glucuronide $(\sim 6.9$ $\min )$. albumin solutions irradiated in a closed system (14). This is in contrast to the Gunn rat in which the configurational isomers are excreted in the bile almost as fast as they are produced in the light-exposed tissues (9) and are nearly undetectable in the serum. The high concentration of configurational isomers found in the serum of human infants indicates that the rate of excretion of these photoproducts is slow compared with their rate of formation. This has been confirmed in a study showing that the mean half-life for the disappearance of the configurational isomers in the serum of premature infants after the end of phototherapy is $15 \mathrm{~h}(10)$.

The results of the present study show that in premature human infants the excretion of lumirubin is rapid; it has a mean apparent half-life of $112 \mathrm{~min}$. This estimate was based on the data from the nine patients in our study and the assumption of first-order kinetics. The data for all the patients was consistent

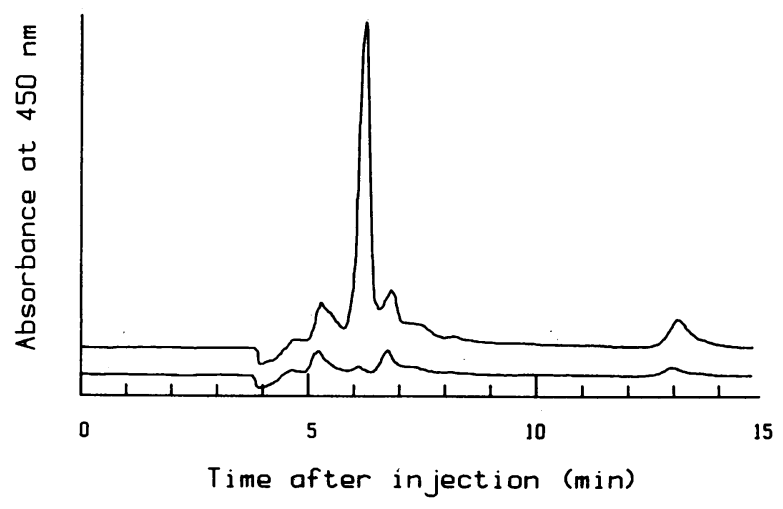

Figure 4. Bilirubin composition in bile of patient number two. Chromatographic analysis was performed on bile obtained during phototherapy (top tracing), and $24 \mathrm{~h}$ after stopping phototherapy (bottom tracing). Both chromatograms are at the same detector attenuation. The peak assignments are the same as in the legend to Fig. 3. 


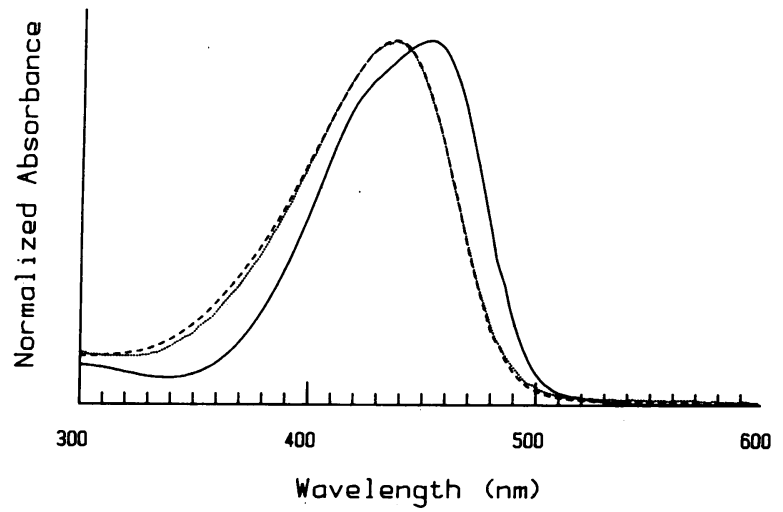

Figure 5. Identification of the principal pigment in the bile during phototherapy as lumirubin. Absorption spectra determined during chromatography are compared for material eluting at $6.2 \mathrm{~min}$ in top chromatogram in Fig. $4(\cdots)$ ), synthetic lumirubin (-- ), and native bilirubin (-). All spectra were normalized to give the same maximal absorption. Absorption spectra of mono- and di-glucuronide conjugates of bilirubin (not shown) have their peak absorption at the same wavelength as native bilirubin.

with a model of lumirubin excretion as a single process with first-order kinetics. However, one of the assumptions of this model, that no lumirubin enters the serum pool after phototherapy stops, is probably not correct. After cessation of phototherapy there is continued migration of lumirubin from its site of formation in light-exposed tissues into the serum. The rate of exchange of lumirubin between this extravascular pool and the serum (intravascular pool), and the size of this extravascular pool of lumirubin, influence how well our measured half-lives reflect the actual rate of lumirubin excretion. If the rate of lumirubin exchange is slow compared with its rate of excretion, then our measured half-life is an accurate measure of lumirubin excretion from the serum. If, however, the rate of lumirubin exchange is fast compared with its rate of excretion, then our measured half-life underestimates the rate of lumirubin excretion. For example, if the exchange were much faster than excretion and the size of the extravascular and serum pools were equal, then the disappearance of lumirubin from the serum would appear to be first order, but the measured serum half-life would be twice the actual half-life. There is indirect evidence that the rate of exchange of lumirubin between the extravascular pool and the serum is comparable with the rate of lumirubin disappearance from the serum (14). Thus, the apparent half-life we have measured may be an overestimate of the actual halflife of lumirubin in the serum. Our results indicate, however, that premature infants are better able to excrete the structural isomer, lumirubin, than the more abundant $4 Z, 15 E$ configurational isomer.

The finding that lumirubin was the major bilirubin species in aspirates of duodenal bile of premature infants under phototherapy and was absent from the bile before (Fig. 3) or after (Fig. 4) phototherapy supports the hypothesis that excretion of lumirubin is more important that excretion of configurational isomers during phototherapy. Onishi and co-workers have also found that lumirubin is the major pigment present in bile obtained from infants receiving phototherapy and is absent before phototherapy $(7,16)$. The bile samples analyzed by these investigators have a higher concentration of bilirubin conjugates than we have found in our samples, suggesting they studied more mature patients. (No information was provided about the patient population in either study by Onishi et al. $[7,16])$. In addition, they report a small increase in the amount of native bilirubin in bile obtained during phototherapy compared with bile obtained before phototherapy (16), although they acknowledge the difficulty of precise quantitation of bile samples. In both our study and those of Onishi and his co-workers $(7,16)$ the conclusion is the same: lumirubin is the principal pigment excreted in the bile of infants treated with phototherapy.

All of the bile samples in our study had small amounts of native, $4 Z, 15 Z$-bilirubin as well as lumirubin. Because native bilirubin is not soluble at the pH of our bile samples $(\sim 7.3)$, it is possible that our analysis has underestimated the amount of native bilirubin actually present in nascent bile. Control experiments have shown that native bilirubin that is not in solution but remains suspended is rapidly solubilized during chromatography and elutes from the column normally. However, some of the native bilirubin present in the bile in situ may have adsorbed to the nasojejunal tubes during sample collection, or to the sides of the tubes in which the bile was stored. Thus, because of the limitations of the way in which bile can be obtained from human infants, we cannot be sure that our analyses reflect the composition of nascent bile. The native bilirubin in the bile is most likely derived principally from excreted configurational isomers that re-isomerize to the more stable native configuration in the bile (9). (Nonenzymatic hydrolysis of bilirubin conjugates may also be a source of native bilirubin [13].) Since native bilirubin is reabsorbed in the proximal small bowel (17), it is possible that the percentage of the total pigment that is initially excreted into the bile as configurational isomers is higher than that which is subsequently found in the aspirate as native bilirubin. Although more configurational isomers may be excreted into bile initially, only that proportion which is not reabsorbed contributes to the net elimination of pigment during phototherapy. The relatively small amount of native bilirubin found in the bile samples is consistent with the slow rate of disappearance of the configurational isomers from the serum (10). Thus, the chromatographic analysis of the bile samples provide further support that in infants treated with phototherapy, formation and excretion of the structural isomer lumirubin is quantitatively more important for pigment elimination than excretion of configurational isomers.

Photooxidation of bilirubin to a variety of colorless monoand di-pyrroles and excretion of these products in the urine also occurs during phototherapy. However, the concentration of these photoproducts in the urine of infants receiving phototherapy (18) is lower than the urinary concentration of lumirubin (16). Since biliary excretion of bilirubin photoproducts is quantitatively more important than urinary excretion (19), it appears that photooxidation is at most a minor contributor to pigment elimination during phototherapy. This is not proven, however, because the chromatographic systems used to analyze bile in this study and in previous studies $(7,16)$ would not detect photooxidation products.

The conclusion from this study, that excretion of lumirubin is quantitatively more important during phototherapy than excretion of configurational isomers, is supported by other recent observations. We have shown (14) that doubling the light intensity used for phototherapy (with no change in color) increased the serum concentration of lumirubin, while leaving unchanged the concentration of configurational isomers. Since higher intensity light leads to more rapid bilirubin elimination (20), these results are consistent with lumirubin excretion being more im- 
portant than configurational isomer excretion. (Although not measured, more bilirubin photooxidation must have also occurred in the infants treated with higher intensity light.) Vecchi and his co-workers have found that fluorescent green light is more effective than broad-spectrum white light in the treatment of neonatal jaundice (21). We have shown that lumirubin concentrations are higher and configurational isomer concentrations are lower in infants treated with green light compared with infants treated with white light (22). Thus, these results also support the conclusion that lumirubin excretion during phototherapy is important. (Based upon the action spectrum for bilirubin photooxidation (23), less photooxidation is expected to occur in infants treated with green light than in infants treated with white light.)

In summary, our findings demonstrate that lumirubin is rapidly cleared from the serum of jaundiced premature infants during phototherapy. Analysis of bile obtained during phototherapy is consistent with lumirubin being the principal bilirubin isomer excreted during phototherapy.

\section{Acknowledgments}

We thank Drs. F. K. Ennever and J. W. Greenberg, and two anonymous reviewers, for helpful comments on the manuscript, and Dr. R. N. Smith for help in obtaining samples of rat bile.

This work was supported by U. S. Public Health Service grants DK-36517, and CA-23692, and RR-00240.

\section{References}

1. Maisels, M. J. 1981. Neonatal jaundice. In Neonatology. G. B. Avery, editor. L.B. Lippincott \& Co., Philadelphia. 473-544.

2. Brown, A. K., M. H. Kim, P. Y. K. Wu, and D. A. Bryla. 1985. Efficacy of phototherapy in prevention and management of neonatal hyperbilirubinemia. Pediatrics. 75:393-400.

3. Lewis, H. M., R. H. A. Campbell, and G. Hambleton. 1982. Use or abuse of phototherapy for physiological jaundice of newborn infants. Lancet. ii:408-410.

4. McDonagh, A. F., L. A. Palma, F. R. Trull, and D. A. Lightner. 1982. Phototherapy for neonatal jaundice: configurational isomers of bilirubin. J. Am. Chem. Soc. 104:6865-6867.

5. McDonagh, A. F., L. A. Palma, and D. A. Lightner. 1982. Phototherapy for neonatal jaundice: Stereospecific and regioselective photoisomerization of bilirubin bound to human serum albumin and NMR characterization of intramolecular cyclized photoproducts. J. Am. Chem. Soc. 104:6867-6869.

6. Stoll, M. S., E. A. Zenone, J. D. Ostrow, and J. E. Zarembo. 1979. Preparation and properties of bilirubin photoisomers. Biochem. J. 183: 139-146.
7. Onishi, S., K. Isobe, S. Itoh, N. Kawade, and S. Sugiyama. 1980. Demonstration of a geometric isomer of bilirubin-IX $\alpha$ in the serum of a hyperbilirubinaemic newborn infant and the mechanism of jaundice phototherapy. Biochem. J. 190:533-536.

8. Lamola, A. A., W. E. Blumberg, R. McClead, and A. Fanaroff. 1981. Photoisomerized bilirubin in blood from infants receiving phototherapy. Proc. Natl. Acad. Sci. USA. 78:1882-1886.

9. McDonagh, A. F. 1984. Molecular mechanisms of phototherapy for neonatal jaundice. In Neonatal Jaundice. F. F. Rubaltelli and G. Jori, editors. Plenum Publishing Corp., New York. 173-185.

10. Ennever, J. F., I. Knox, S. C. Denne, and W. T. Speck. 1985. Phototherapy for neonatal jaundice: In vivo clearance of bilirubin photoproducts. Pediatr. Res. 19:205-208.

11. Ennever, J. F., M. Sobel, A. F. McDonagh, and W. T. Speck. 1984. Phototherapy for neonatal jaundice: In vitro comparison of light sources. Pediatr. Res. 18:667-670.

12. Harvey, M. C., and S. D. Stearns. 1983. HPLC sample injection using electric valve actuators. J. Chromatogr. Sci. 21:473-477.

13. McDonagh, A. F., L. A. Palma, J. J. Lauff, and T.-W. Wu. 1984. Origin of mammalian biliprotein and rearrangement of bilirubin glucuronides in vivo in the rat. J. Clin. Invest. 74:763-770.

14. Costarino, A. T., J. F. Ennever, S. Baumgart, W. T. Speck, M. Paul, and R. A. Polin. 1985. Bilirubin photoisomerization in premature neonates under low- and high-dose phototherapy. Pediatrics. 75:519522.

15. Malhotra, V., and J. F. Ennever. 1986. Determination of the relative detector response for unstable bilirubin photoproducts without isolation. J. Chromatog. 383:153-157.

16. Onishi, S., K. Isobe, S. Itoh. 1986. Metabolism of bilirubin and its photoisomers in newborn infants during phototherapy. J. Biochem. (Tokyo). 100:789-795.

17. Lester, R., and R. Schmid. 1963. Intestinal absorption of bile pigments. I. The enterohepatic circulation of bilirubin in the rat. J. Clin. Invest. 42:736-746.

18. Lightner, D. A., W. P. Linnane III, and C. E. Ahlfors. 1984. Bilirubin photooxidation products in the urine of jaundiced infants receiving phototherapy. Pediatr. Res. 18:696-700.

19. Callahan, E. W., M. M. Thaler, M. Karon, K. Bauer, and R. Schmid. 1970. Phototherapy of severe unconjugated hyperbilirubinemia: Formation and removal of labeled bilirubin derivatives. Pediatrics. 46: 841-848.

20. Tan, K. L. 1977. The nature of the dose-response relationship of phototherapy for neonatal hyperbilirubinemia. J. Pediatr. 90:448452.

21. Vecchi, C., G. P. Donzelli, M. G. Migliorini, and G. Sbrana. 1983. Green light in phototherapy. Pediatr. Res. 17:461-463.

22. Ennever, J. F., I. Knox, and W. T. Speck. 1986. Differences in bilirubin isomer composition in infants treated with green and white light phototherapy. J. Pediatr. 109:119-122.

23. Lightner, D. A., T. A. Wooldridge, S. L. Rodgers, and R. D. Norris. 1980. Action spectra for bilirubin photodisappearance. Experientia. 36:380-382. 